\title{
V346 Centauri: Early-type eclipsing binary with apsidal motion and abrupt change of orbital period $\star, \star \star$
}

\author{
Pavel Mayer ${ }^{1}$, Petr Harmanec ${ }^{1}$, Marek Wolf ${ }^{1}$, Jana Nemravová ${ }^{1}$, Andrej Prša ${ }^{2}$, Yves Frémat ${ }^{3}$, Miloslav Zejda ${ }^{4}$, \\ Jiři Liška ${ }^{4}$, Jakub Juryšek ${ }^{1,5,6}$, Kateřina Hoňková ${ }^{6}$, and Martin Mašek ${ }^{5,6}$ \\ 1 Astronomical Institute of the Charles University, Faculty of Mathematics and Physics, V Holešovičkách 2, \\ 18000 Praha 8, Czech Republic \\ e-mail: mayer@cesnet.cz \\ 2 Department of Astrophysics and Planetary Sciences, Villanova University, 800 East Lancaster Ave, Villanova, \\ PA 19085, USA \\ 3 Royal Observatory of Belgium, Ringlaan 3, 1180 Brussels, Belgium \\ ${ }^{4}$ Department of Theoretical Physics and Astrophysics, Masaryk University, Kotlářská 2, 61137 Brno, Czech Republic \\ 5 Institute of Physics, The Czech Academy of Sciences, Na Slovance 1999/2, 18221 Praha 8, Czech Republic \\ 6 Variable Star and Exoplanet Section of the Czech Astronomical Society, Vsetínská 941/78, 75701 Valašské Meziříčí, \\ Czech Republic
}

Received 7 April 2016 / Accepted 2 May 2016

\begin{abstract}
New physical elements of the early B-type eclipsing binary V346 Cen are derived using the HARPS spectra downloaded from the ESO archive and also numerous photometric observations from various sources. A model of the observed times of primary and secondary minima that fits them best is a combination of the apsidal motion and an abrupt decrease in the orbital period from 6.322123 to 6.321843 (shortening by $24 \mathrm{~s}$ ), which occurred somewhere around JD 2439000 . Assumption of a secularly decreasing orbital period provides a significantly worse fit. Local times of minima and the final solution of the light curve were obtained with the program PHOEBE. Radial velocities of both binary components, free of line blending, were derived via $2 \mathrm{D}$ cross-correlation with a program built on the principles of the program TODCOR. The oxygen lines in the secondary spectra are weaker than those in the model spectra of solar chemical composition. Using the component spectra disentangled with the program KOREL, we find that both components rotate considerably faster than would correspond to the synchronization at periastron. The apside rotation known from earlier studies is confirmed and compared to the theoretical value.
\end{abstract}

Key words. stars: early-type - binaries: close - stars: individual: V346 Cen

\section{Introduction}

The eclipsing binary V346 Cen (HD 101837; $V=8^{\mathrm{m}} .3$ in maximum, with a period of 6.322 ) has an eccentric orbit with $e=0.29$ and is one of the early-type binaries for which the apside line rotation has been found. Its variability was discovered by O'Leary \& O'Connell (1936) and the presence of apsidal motion was first reported by Dugan \& Wright (1939) and O'Connell (1939). Although the star was classified as B5 on a slit spectrogram by Popper (1966), the standard MK spectral type appears to be B0.5 IV according to Fitzgerald \& Miller (1983). The binary is a member of the open cluster Stock 14.

Several photometric and spectroscopic studies of V346 Cen have been carried out, the fundamental paper being by Giménez et al. (1986a, GCA). They obtained the apside line rotation period of $321 \pm 16$ years. An important contribution has also been presented by Drobek et al. (2013); they give the apsidal period as $306 \pm 4 \mathrm{yr}$. New photometric and spectroscopic data

\footnotetext{
* Based on observations made with the ESO telescopes at the La Silla Paranal Observatory under programmes ID 083.D-0040(A), 085.C0614(A), and 178.D-0361(B).

$\star \star$ Tables A.2-A.6 are only available at the CDS via anonymous ftp to cdsarc.u-strasbg. fr $(130.79 .128 .5)$ or via

http://cdsarc.u-strasbg.fr/viz-bin/qcat?]/A+A/591/A129
}

have been published since then and we have secured several sets of new photometric observations.

\section{Photometry}

New photometric observations have been published by van Houten et al. (2009), by the ASAS 2 and ASAS 3 project teams (Pojmański 1998, 2002), and by Drobek et al. (2013). We used the photographic light curve of O'Connell (1939) and obtained new $B V R, B V R I$, and non-stardard green photometry at three different stations. The journal of photometric observations used here is in Table $1^{1}$. Details on the individual data sets and tables with new observations can be found in the Appendix. We note that one of the comparison stars we used at the Mount John Observatory, and which was also used by O'Connell (1939) and van Houten et al. (2009), is an irregular B0.5 e variable V916 Cen that should be abandoned as a comparison in any future observations (see Fig. B.1). Pojmański (2000) and several other investigators reported that the star is an eclipsing binary with a period of 1.463 , but we argue that it is a typical Be variable showing the variations on several time scales (see Appendix B for details). Obviously, the van Houten et al. (2009)

\footnotetext{
1 Throughout this study we will use the following abbreviation for the
} reduced Julian date RJD = HJD-2 400000. 
Table 1. Journal of photometric observations of V346 Cen.

\begin{tabular}{ccclc}
\hline \hline Source & $\begin{array}{c}\text { Time interval } \\
\text { RJD }\end{array}$ & $\begin{array}{c}\text { No. of } \\
\text { obs. }\end{array}$ & $\begin{array}{l}\text { Photometric } \\
\text { system }\end{array}$ & $\begin{array}{c}\text { Comparison } \\
\text { /check }\end{array}$ \\
\hline 1 & $26828.0-27006.3$ & 287 & photographic \\
2 & $42157.3-42236.3$ & $76 / 75 / 74 / 75$ & VBUL Walraven & HD 101794/HD 101838 \\
3 & $44991.7-45459.8$ & 1056 & uvby & HD 101466/HD 100942 \\
4 & $50547.9-50583.8$ & 192 & $I$ & all-sky \\
4 & $51111.9-51197.8$ & 75 & $I$ & all-sky \\
4 & $51200.6-51403.5$ & 177 & $I$ & all-sky \\
4 & $51475.9-51562.8$ & 104 & $I$ & all-sky \\
5 & $51899.8-53497.8$ & 252 & $V$ & all-sky \\
5 & $53503.6-55048.5$ & 251 & $V$ & all-sky \\
6 & $54117.9-54120.1$ & $362 / 322 / 283$ & $B V R$ & HD 101794/- \\
7 & $54167.1-54234.0$ & $833 / 1048 / 1078$ & $U B V$ & several \\
8 & $55692.3-55703.4$ & 1567 & green & HD 102152/HD 308 \\
9 & $56680.6-56842.7$ & $1273 / 1353 / 1478 / 1485$ & $B V R I$ & CD-61 $3157 /$ CPD-61 2540 \\
\hline
\end{tabular}

Notes. $\left.{ }^{*}\right)$ Compared with the sequence of HD 101511, HD 101838, HD 101794, and HD 102053. Column “Source”: 1. O'Connell (1939), Riverview photographic plates; 2. van Houten et al. (2009), $0.90 \mathrm{~m}$ Dutch telescope, Leiden Southern Station, BroederStroom, South Africa; 3. Giménez et al. (1986b), $0.50 \mathrm{~m}$ Danish reflector, ESO, La Silla, Chile; 4. Pojmański (1998, 2000), ASAS 2: $0.135 \mathrm{~m}$ telephoto lens with a Meade CCD camera, Las Campanas, Chile; 5. Pojmański (2002), ASAS 3: 0.200 m Minolta telephoto lens with a CCD camera, Las Campanas, Chile; 6. this paper, $0.6 \mathrm{~m}$ reflector with a CCD camera, Mt. John, New Zealand; 7. Drobek et al. (2013), Australian National University $1.0 \mathrm{~m}$ reflector, Wide Field Imager of eight CCD chips, Siding Springs, Australia; 8. this paper, Sonnar 4/135 mm telephoto lens with a CCD ATIK16IC camera, Sutherland, South Africa; 9. this paper, 0.3 m Meade Schmidt-Cassegrain reflector with a CCD camera (Ebr et al. 2014).

and our Mt. John observations should be treated with some caution.

\subsection{Times of minima}

The first times of minima were published by the discoverers of the variability O'Leary \& O'Connell (1936), O'Connell (1939), and Dugan \& Wright (1939). When Hernandez \& Sahade (1978) obtained the first radial-velocity (RV) curve of the primary component of V346 Cen, it turned out that Dugan \& Wright (1939) had incorrectly identified the primary and secondary minima. Their identification is correct in O'Connell (1939). Van Houten (2009) obtained the light curve in four passbands of the Walraven system and also published a time of a primary minimum. The star was not observed by HIPPARCOS, and the TYCHO data are too noisy to be used to determine the times of minima. The time of the primary minimum RJD 50554.442 was obtained from the ASAS 2 observations by Pojmański (1998) (although his period of $6.295 \mathrm{~d}$ is rather off). Ogloza \& Zakrzewski (2004) have published several times of minima based on ASAS 2 photometry, but their primary and secondary minima are also reversed. Drobek et al. (2013) published several times of minima, based once more on the ASAS 2, on ASAS 3 (Pojmański 2002), van Houten et al. (2009), and their new observations.

Here, we adopt a different approach. Considering that the orbital period is rather long for an accurate determination of individual minima, and having all the light curves at our disposal, we fitted all individual local light curves in individual passbands, covering time segments not longer than $1500 \mathrm{~d}$ (and usually much shorter - see the time intervals specified in Table 1) using the PHOEBE program (Prša \& Zwitter 2005, 2006) rel. 1.0 with several passbands added. In this way, we kept most of the elements fixed at the values of our final solution (see Sect. 4) and we only allowed convergency of the phase shift from a local mean epoch, local value of the longitude of periastron, and the light level. This way, we obtained the local epochs of the primary and secondary minima. A few examples of the light-curve fits can be found in Fig. A.1.

For completeness we mention that there is also a photographic light curve by Gaposchkin (1953) constructed from estimates on plates of the Harvard collection obtained in the years 1941-1950. The light curve is published only in the form of 28 normal points, which inevitably smears out the effects of the apsidal motion. For this reason, we have not considered this data in our analyses.

Dugan \& Wright (1939) published only the times of minima, not the individual magnitude estimates. Since they mention that they applied the heliocentric corrections of time, we tried to reconstruct the accurate times of minima from their Table 3, using their epochs and the $\mathrm{O}-\mathrm{C}$ tabulated separately in days, and in orbital phases. This gave HJDs, which never differed for more than $0.003 \mathrm{~d}$ and we adopted the mean of these two estimates. All these reconstructed Dugan \& Wright (1939) minima are summarized in Table A.1. The minima based on local fits of the light curves are listed in Table 2. They are complemented by two estimates of local minima based on our RV solutions for the Hernandez \& Sahade (1978), and our new RVs (see below).

Following the procedure already applied by Dugan \& Wright (1939), we derived two straight lines via least-squares fits in the $\mathrm{O}-\mathrm{C}$ residuals and taking the weights of individual minima into account. In doing so we omitted (as also advised by Dugan \& Wright 1939) the first three primary minima, and the first secondary minimum. From the intersection of both straight lines thus constructed we estimated the instant when the longitude of periastron was $\omega=270^{\circ}$ as RJD 21128 . Dugan \& Wright (1939) gives this time as RJD 21963, Drobek et al. (2013) as 20750. These numbers probably characterize the uncertainty of this determination. 


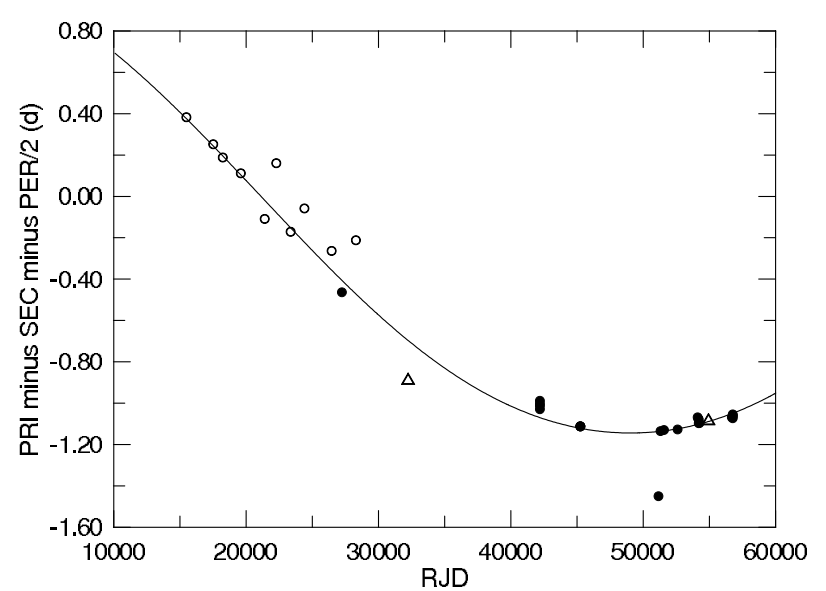

Fig. 1. Differences of times of minima from Table A.1 (open circles) and Table 2 (filled circles; triangles when the time results from a RV curve) are shown. Some data, probably of low accuracy, differ from the dominant trend. The curve corresponds to the period of apsidal advance 306 years and parameters in Tables 3 and 5 .

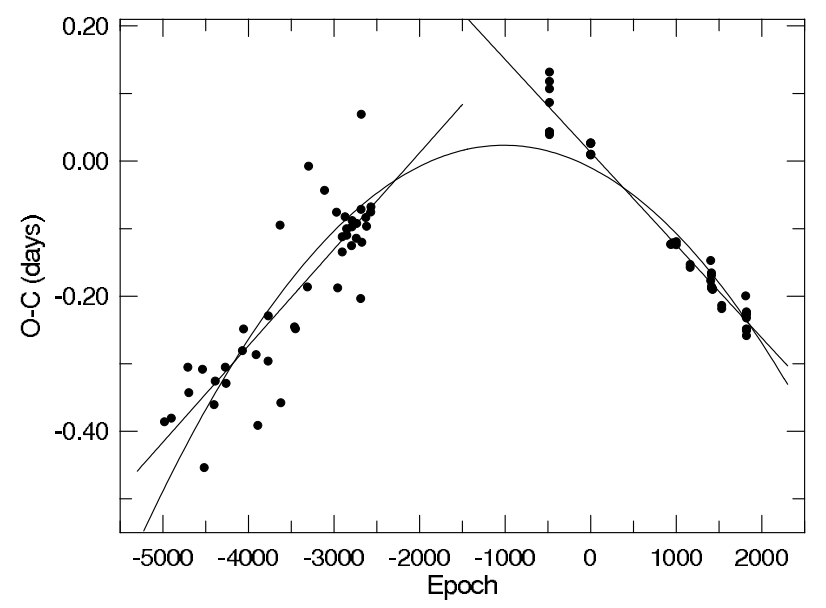

Fig. 2. Time plot of the $\mathrm{O}-\mathrm{C}$ deviations from the fit of the apsidalmotion curves to the minima of V346 Cen. The asymmetry of the plot depends on $P_{\text {sider }}$ used; here it is 6.32198 .

The differences between the times of the primary and secondary minima at a given epoch depend on the rate of the apside rotation alone (see e.g. Giménez \& Garcia-Pelayo 1983; Khaliullina et al. 1985; Giménez \& Bastero 1995). When Drobek et al. (2013) carried out the analysis of the apsidal motion, they also found a clear deviations from a pure apsidal motion (cf. their Fig. 14). They tentatively suggested that these deviations could be due to the light-time effect caused by the presence of a third body in the system with an orbital period longer than $100 \mathrm{yr}$.

Because we had more observations than Drobek et al. (2013) at our disposal, we repeated their analysis, using the primary and secondary minima from Tables A.1 and 2; the result is shown in Fig. 1. A somewhat better fit than theirs was obtained when we used our value RJD = 21 128 for the epoch, when $\omega=270^{\circ}$; the period of the apsidal advance of 306 years does not need any revision.

Our plot of $\mathrm{O}-\mathrm{C}$ deviations from the model apsidal motion is in Fig. 2. It would be difficult to explain it as an effect of a third body since, for example, the shape of the plot of deviations is not that of a sine-type curve expected for the light-time effect. With the period about 200 years and semi-amplitude $\approx 0.25$ the
Table 2. Times of the primary and secondary minima of V346 Cen estimated from locally fitted individual light curves as defined in Table 1 (see text for details).

\begin{tabular}{llll}
\hline \hline Min I & Min II & rms & Source \\
RJD & RJD [d] & & \\
\hline 27226.5715 & 27223.8744 & 0.0071 & phot. O'Connell \\
32239.68 & 32237.40 & 0.13 & RVs ${ }^{1}$ \\
42196.943 & 42194.795 & 0.012 & Walraven $V$ \\
42196.954 & 42194.795 & 0.015 & Walraven $B$ \\
42196.923 & 42194.791 & 0.017 & Walraven $U$ \\
42196.968 & 42194.795 & 0.014 & Walraven $L$ \\
45250.3726 & 45248.3227 & 0.0005 & Stromgren $y$ \\
45250.3734 & 45248.3235 & 0.0005 & Stromgren $b$ \\
45250.3729 & 45248.3235 & 0.0004 & Stromgren $v$ \\
45250.3727 & 45248.3238 & 0.0005 & Stromgren $u$ \\
51161.311 & 51159.288 & 0.010 & ASAS2 \\
51306.7203 & 51304.6942 & 0.0044 & ASAS2 \\
51572.2482 & 51570.2167 & 0.0048 & ASAS2 \\
52602.7051 & 52600.6705 & 0.0032 & ASAS3 \\
54120.0110 & 54117.9181 & 0.0027 & Mt. John $R$ \\
54176.8914 & 54174.8069 & 0.0013 & $V^{2}$ \\
54176.8881 & 54174.8046 & 0.0015 & $B^{2}$ \\
54202.158 & 54200.093 & 0.026 & Siding Spring $U$ \\
54252.7317 & 54250.6666 & 0.0028 & ASAS3 \\
54929.1703 & 54927.0861 & 0.0093 & RV TODCOR \\
56699.3383 & 56697.2455 & 0.0020 & SAAO green \\
56762.5371 & 56760.4400 & 0.0006 & FRAM $V$ \\
56762.5296 & 56760.4410 & 0.0014 & FRAM $B$ \\
56762.5390 & 56760.4368 & 0.0007 & FRAM $R$ \\
56762.5396 & 56760.4320 & 0.0007 & FRAM $I$ \\
\hline
\end{tabular}

Notes. ${ }^{(1)}$ Hernandez \& Sahade (1978). ${ }^{(2)}$ Mt. John \& Sidding Spring.

minimum mass of the third body (for the masses of the eclipsing binary components according to Table 5) would have to be more than $\approx 10 M_{\odot}$. No such body with the corresponding luminosity is detectable in either the light curves or the high signalto-noise $(\mathrm{S} / \mathrm{N})$ spectra, as detailed below. Even if we assume that the third body is a binary, its light and spectral lines should be visible (and the body should be observable by speckle interferometry as the separation should be of the order of tens of mas).

One possible explanation could be a continuous secular change in the period, shown in Fig. 2 by a parabola with $P=$ 6.321915 and with the second order term $-3.22 \times 10^{-8} \times E^{2}$. This fit could be improved if a combination with the light-time effect by a third body were considered. The light-time effect amplitude might then be small and there would be no problem with the absence of the signatures of the third body in the spectra and photometric solutions.

As Fig. 2 shows, the assumption of a sudden decrease in the sidereal period near RJD 39000 fits the observations much better. If the decrease occurred, the sidereal period was 6.322123 prior to RJD 39000 (similar to 6.322070 advocated by O'Connell 1939) and 6.321843 after RJD 39000 . The change is 1:1.000044 (a decrease of $24 \mathrm{~s}$ ) and it occurred during an interval of about a $30 \mathrm{yr}$ for which no observations of eclipses are available. A similar period decrease happened in the V505 Sgr three-body system, and Brož et al. (2010) tentatively explained it by a close encounter with a body on hyperbolic orbit. A sudden period change was observed for several other binaries, e.g. TW Dra (Zejda et al. 2008) and UV Leo (Mikuz et al. 2002); 


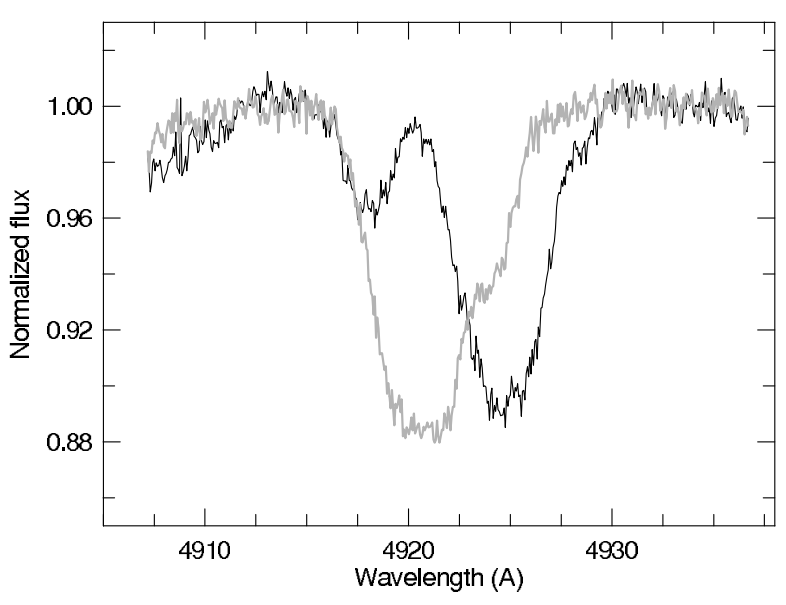

Fig. 3. He I $4922 \AA$ line in the spectra of extreme velocities (black line: RJD 54 928.50, grey line: RJD 54930.81 ). The line is blended with O II $4924.52 \AA$ and is affected by the O II $4906.82 \AA$ line at the blue side.

in these cases, periods increased. Moreover, TW Dra is a semidetached system, where an episode of more intense mass transfer would not be so surprising.

For the moment, we have no clear explanation for either the continuous or sudden period change of V346 Cen; judging by a normal appearance of the $\mathrm{H} \alpha$ line, without any detectable trace of emission indicative of the presence of some circumstellar matter in the system, we do not find the possibility of a transient mass transfer event very probable, though we admit that we do not have any spectra taken around RJD 39000.

\section{Spectroscopy}

At present, there are 33 spectra in the ESO archive taken by the echelle spectrograph HARPS at the $3.6 \mathrm{~m}$ ESO telescope. These spectra were obtained during a 6-day interval in April 2009 and are available as a pipeline product. The list of spectra is in Table 4. The spectra cover the region from 378 to $691 \mathrm{~nm}$ and have the resolving power of 115000 ; we binned the spectra to the resolution $0.06 \AA$, typically obtaining a S/N of $\sim 250$. Fringing is apparent at wavelengths longer than $450 \mathrm{~nm}$. Examples of the He I $4922 \AA$ line near two opposite elongations are in Fig. 3.

\subsection{Radial velocities}

As already mentioned, the first RV curve of the primary component V346 Cen was obtained by Hernandez \& Sahade (1978), but it seems that this curve was affected by the line blending of the primary and secondary. The authors themselves noted that the high eccentricity of $0.40 \pm 0.02$ they obtained is suspect. Our experience from other similar systems is, however, that their RVs can still be used to determine local epochs of the primary and secondary minima if the correct value of eccentricity is fixed in the solution. Using the program FOTEL (Hadrava 2004a), we therefore derived a new solution for their primary RVs, keeping the sidereal period fixed at our value of 6.322123 and the eccentricity at a value of 0.287 , which follows from new RVs and light curves (see below). The solution is given in Table 3 .

Two $20 \AA \mathrm{mm}^{-1}$ fine-grained photographic spectra at both elongations were obtained by GCA, who estimated $K_{1}=135 \pm$ 5 , and $K_{2}=190 \pm 10 \mathrm{~km} \mathrm{~s}^{-1}$. Clearly, new and more complete $\mathrm{RV}$ curves of both components are desirable.
Table 3. Orbital solutions for the RVs of the primary obtained by Hernandez \& Sahade (1978), and for our new HARPS RVs derived with asTODCOR.

\begin{tabular}{lrr}
\hline \hline Element & RVs HS & HARPS RVs \\
\hline$P_{\text {sider. }}[\mathrm{d}]$ & 6.322123 fixed & 6.321843 fixed \\
$T_{0}[\mathrm{RJD}]$ & $32237.88(13)$ & $54928.4445(93)$ \\
$T_{\text {min.I }}[\mathrm{RJD}]$ & 32239.88 & 54929.1703 \\
$T_{\text {min.II }}[\mathrm{RJD}]$ & 32237.40 & 54927.0861 \\
$e$ & 0.287 fixed & 0.287 fixed \\
$\omega\left[^{\circ}\right]$ & $319.1(8.5)$ & $20.29(68)$ \\
$K_{1} / K_{2}$ & - & $0.7121(42)$ \\
$K_{1}\left[\mathrm{~km} \mathrm{~s}^{-1}\right]$ & $107.2(8.1)$ & $136.46(43)$ \\
$K_{2}\left[\mathrm{~km} \mathrm{~s}^{-1}\right]$ & - & $191.65(1.29)$ \\
$\mathrm{rms}_{1}\left[\mathrm{~km} \mathrm{~s}^{-1}\right]$ & 18.4 & 1.54 \\
$\mathrm{rms}_{2}\left[\mathrm{~km} \mathrm{~s}^{-1}\right]$ & - & 3.66 \\
\hline
\end{tabular}

Notes. The use of disentangled template spectra leads formally to zero systemic velocities for both components; by comparing the disentangled spectra to rotationally broadened synthetic spectra, we estimated the true systemic velocity of V 346 Cen to be $-4 \pm 3 \mathrm{~km} \mathrm{~s}^{-1}$. For comparison, GCA obtained $-8 \pm 3 \mathrm{~km} \mathrm{~s}^{-1}$ from their two photographic spectra taken in opposite elongations.

Determination of RVs by direct measurements of individual lines (e.g. using Gaussians) in the HARPS spectra is not a good method for V346 Cen. The lines are rotationally broadened and nearly all stronger lines (mainly $\mathrm{He} \mathrm{I}$ ) are accompanied by numerous blends, most often by $\mathrm{O}$ II lines, which prevents reliable measurements. In another attempt, we tried to disentangle the spectra using the program KOREL (Hadrava 2004b) in several spectral regions. The spread of the results was larger than expected. Nevertheless, we formed a mean of the KOREL solutions from six spectral regions and used it as a starting solution for spectra disentangling. A relatively large spread in RVs was also found when we tried to obtain them from the comparison of individual spectra with two synthetic spectra.

Therefore, we finally used the program asTODCOR ${ }^{2}$ based on the principles of a 2D cross-correlation approach, first suggested by Zucker \& Mazeh (1994) - see Desmet et al. (2010) for the details of program asTODCOR. Following Harmanec et al. (2015) we adopted the spectra disentangled by KOREL as the templates for the 2D cross-correlation. The spectral region $4460-4486 \AA$ was used.

The orbital solution based on asTODCOR RVs had small rms errors, and actually agrees very well with the mean KOREL solution. The asTODCOR RVs are listed in Table 4, the RV curves are in Fig. 4, and the orbital solution for the fixed values of $P_{\text {sider. }}$ and $e$ are also listed in Table 3 .

\subsection{A negative search for the tertiary}

We carried out several experiments with KOREL to check whether a line spectrum of a putative tertiary could not be found in the high-quality HARPS spectra. Because all HARPS spectra were obtained within a short interval of 6 days, it is clear that the exact value of the long orbital period (more than some $100 \mathrm{yr}$, see Sect. 2.1) used for the disentangling is not important. We ran a number of trial solutions with KOREL, but in no case were any lines of a tertiary found.

\footnotetext{
2 Written by Y.F.
} 


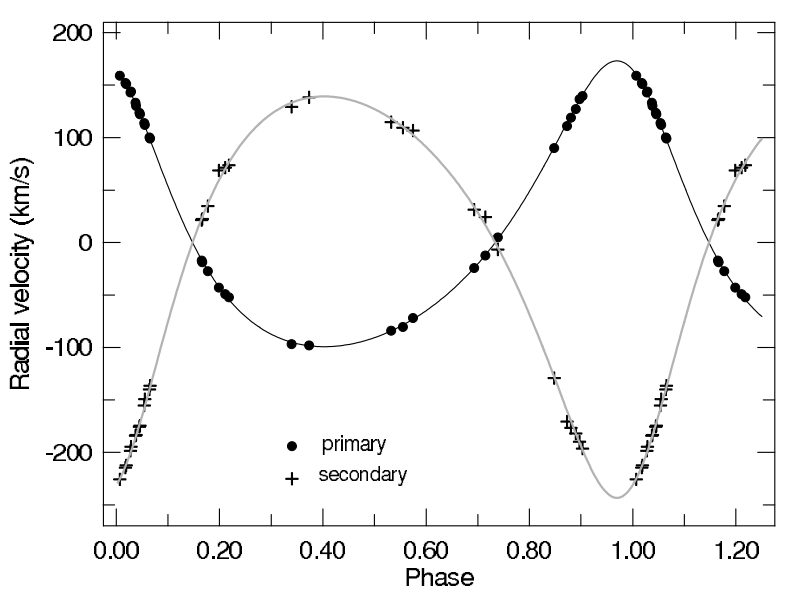

Fig. 4. Radial velocities of the primary and secondary components of V346 Cen as calculated by asTODCOR.

\subsection{Comparison with synthetic spectra}

We tried to support the spectral classification of the binary components via a comparison of the disentangled and synthetic spectra. The spectra were disentangled by KOREL for the fixed orbital elements given by the HARPS solution of Table 3 . We used a Python program PYTERPOL, which interpolates in a precalculated grid of synthetic spectra in order to estimate radiative properties of components of multiple systems ${ }^{3}$. We used it to fit the disentangled spectra with the synthetic BSTAR spectra (Lanz \& Hubeny 2007). Solar metallicity and a microturbulence of $2 \mathrm{~km} \mathrm{~s}^{-1}$ were assumed; the $\log g$ values were fixed from the joint light-curve and RV curve solution (see Sect. 4). Formally, the best fit temperatures were $T_{\text {eff }, 1}=27141 \pm 25 \mathrm{~K}$ and $T_{\text {eff }, 2}=20991 \pm 190 \mathrm{~K}$. The comparison also provided rotational velocities of both components (see Table 5 ). As already noted by GCA, who obtained values of $165 \pm 15$ and $140 \pm 15 \mathrm{~km} \mathrm{~s}^{-1}$, these rotation velocities are considerably higher than those corresponding to (pseudo)synchronization (see Table 5).

The effective temperature of the secondary obtained this way is much lower than that derived from the light-curve solution (Sect. 4). It seems that the reason is the weakness of the $\mathrm{O}$ II lines in the secondary spectrum. A part of the disentangled spectrum with several O II lines is shown in Fig. 5. When we compared the disentangled spectra of the secondary with a synthetic spectrum for $T_{\text {eff }}=25000 \mathrm{~K}$ obtained from the light-curve solution for He I and some other lines, we found a very good agreement. Even a direct comparison of the $21000 \mathrm{~K}$ and $25000 \mathrm{~K}$ synthetic spectra for $\log g=4.0$ [cgs] rotated to $93 \mathrm{~km} \mathrm{~s}^{-1}$ shows their close similarity for the majority of spectral features in the optical part of the spectrum. We therefore believe that there is no real discrepancy, but only a mild underabundancy of $\mathrm{O}$ II in the atmosphere of the secondary.

\subsection{Spectral type of the secondary}

The spectral type and luminosity class of the primary noted in the Introduction is accepted here; according to Schmidt-Kaler (in Aller et al. 1982), the temperature $T_{\text {eff }, 1}$ is then $\approx 27000 \mathrm{~K}$. The secondary can be classified using the criteria listed by Lesh (1968): as the line O II $4072 \AA$ is not present in the secondary

\footnotetext{
3 A description of the program, developed by J.N., together with a tutorial is available at:

https://github.com/chrysante87/pyterpol/wiki
}

Table 4. HARPS spectra and RVs derived with asTODCOR.

\begin{tabular}{|c|c|c|c|c|c|}
\hline RJD & Phase & Pri RV & $\mathrm{O}-\mathrm{C}$ & Sec RV & $\mathrm{O}-\mathrm{C}$ \\
\hline 54925.4951 & 0.5320 & -84.1 & 0.7 & 115.8 & -4.0 \\
\hline 54925.6391 & 0.5548 & -80.5 & -1.3 & 110.6 & -1.3 \\
\hline 54925.7629 & 0.5743 & -72.0 & 1.6 & 108.0 & 4.0 \\
\hline 54926.5111 & 0.6927 & -24.5 & -0.2 & 32.5 & -2.3 \\
\hline 54926.6485 & 0.7144 & -12.6 & -0.7 & 25.4 & 8.0 \\
\hline 54926.8012 & 0.7386 & 4.7 & 1.3 & 1.1 & -1.5 \\
\hline 54927.4915 & 0.8477 & 89.9 & -2.0 & -121.4 & 0.2 \\
\hline 54927.6494 & 0.8727 & 111.1 & -3.7 & -162.8 & -9.0 \\
\hline 54927.6958 & 0.8800 & 118.9 & -2.6 & -168.9 & -5.6 \\
\hline 54927.7561 & 0.8896 & 127.1 & -3.2 & -174.3 & 1.4 \\
\hline 54927.8018 & 0.8968 & 136.6 & 0.4 & -182.1 & 1.9 \\
\hline 54927.8369 & 0.9024 & 139.7 & -1.2 & -188.6 & 1.9 \\
\hline 54928.4966 & 0.0067 & 158.9 & -0.9 & -218.0 & -0.9 \\
\hline 54928.5656 & 0.0176 & 152.1 & 1.0 & -206.7 & -1.9 \\
\hline 54928.5729 & 0.0188 & 150.9 & 0.9 & -204.6 & -1.3 \\
\hline 54928.6262 & 0.0272 & 143.1 & 0.6 & -190.8 & 1.9 \\
\hline 54928.6335 & 0.0284 & 143.9 & 2.7 & -186.9 & 4.1 \\
\hline 54928.6881 & 0.0370 & 133.2 & 1.1 & -176.4 & 1.7 \\
\hline 54928.6955 & 0.0382 & 130.2 & -0.5 & -175.7 & 0.5 \\
\hline 54928.7366 & 0.0447 & 123.5 & 0.7 & -167.9 & -2.6 \\
\hline 54928.7439 & 0.0458 & 122.1 & 0.7 & -166.7 & -3.6 \\
\hline 54928.7955 & 0.0540 & 113.8 & 2.1 & -147.6 & 1.9 \\
\hline 54928.8028 & 0.0552 & 112.0 & 1.8 & -141.4 & 6.0 \\
\hline 54928.8595 & 0.0641 & 100.0 & 0.7 & -132.3 & -0.2 \\
\hline 54928.8668 & 0.0653 & 99.1 & 1.4 & -128.6 & 1.2 \\
\hline 54929.4982 & 0.1651 & -17.3 & -0.8 & 22.5 & -1.4 \\
\hline 54929.5055 & 0.1663 & -19.0 & -1.4 & 23.6 & -1.7 \\
\hline 54929.5751 & 0.1773 & -27.5 & -1.1 & 35.8 & -1.9 \\
\hline 54929.7096 & 0.1986 & -43.1 & -0.7 & 69.8 & 9.5 \\
\hline 54929.7866 & 0.2108 & -49.4 & 0.7 & 72.6 & 1.6 \\
\hline 54929.8322 & 0.2180 & -52.3 & 2.2 & 74.8 & -2.4 \\
\hline 54930.5976 & 0.3390 & -97.0 & -1.5 & 130.5 & -4.3 \\
\hline 54930.8134 & 0.3732 & -98.1 & 0.9 & 139.6 & -0.1 \\
\hline
\end{tabular}

Notes. The $\mathrm{O}-\mathrm{C}$ residuals are from our FOTEL solution of Table 3. RVs and $\mathrm{O}-\mathrm{C}$ are in $\mathrm{km} \mathrm{s}^{-1}$.

spectrum, the type is B2; and since O II $4121 \AA$ is weaker than He I $4144 \AA$, the luminosity is V. We see that the mass and radius agree with expectations for such a classification.

\section{Final light-curve and RV curve solution}

The excellent uvby light curves published by Giménez et al. (1986b, GCHV) are still the best light curves available for V346 Cen. Each of them is formed by 1056 individual observations obtained in the years 1982 and 1983. As yet another check on the possible presence of a third light, we first run a PHOEBE solution in which third light was among the free parameters of the solution. It resulted in finding zero third light as the optimal result.

To derive the final solution we therefore used the $u v b y$ observations together with the asTORCOR RVs from the HARPS spectra and for the sidereal period derived in the previous section. We fixed the gravity darkening and albedos of both components at 1.0 and the limb-darkening coefficients were interpolated in the grid of pre-calculated model atmospheres. The effective temperature of the primary was kept fixed at $T_{\text {eff, } 1}=$ $27141 \mathrm{~K}$. The theoretical $u$ light curve is compared with the measurements in Fig. 6. 
Table 5. Combined radial-velocity curve and light-curve solution with PHOEBE.

\begin{tabular}{|c|c|c|c|c|}
\hline Element & \multicolumn{4}{|c|}{ Orbital properties } \\
\hline$P_{\text {sider. }}[\mathrm{d}]$ & \multicolumn{4}{|c|}{6.321843 fixed } \\
\hline & \multicolumn{4}{|c|}{0.287 fixed } \\
\hline$\omega$ [degrees] & \multicolumn{4}{|c|}{$347.425 \pm 0.048$} \\
\hline$T_{\text {periast }}[\mathrm{RJD}]$ & \multicolumn{4}{|c|}{45249.14797} \\
\hline$T_{\text {sup.c. }}[\mathrm{RJD}]$ & \multicolumn{4}{|c|}{$45250.36603 \pm 0.00018$} \\
\hline$T_{\text {inf.c. }}[\mathrm{RJD}]$ & \multicolumn{4}{|c|}{45248.31966} \\
\hline$M_{2} / M_{1}$ & \multicolumn{4}{|c|}{$0.7061 \pm 0024$} \\
\hline$i$ [degrees] & \multicolumn{4}{|c|}{$84.423 \pm 0.017$} \\
\hline \multirow[t]{3}{*}{$a\left[R_{\odot}\right]$} & \multicolumn{4}{|c|}{$39.358 \pm 0.094$} \\
\hline & \multicolumn{4}{|c|}{ Component properties } \\
\hline & \multicolumn{2}{|c|}{ primary } & \multicolumn{2}{|c|}{ secondary } \\
\hline$T_{\text {eff }}[\mathrm{K}]$ & \multicolumn{2}{|c|}{27141 fixed } & \multicolumn{2}{|c|}{$25376 \pm 18$} \\
\hline$F^{*}$ & \multicolumn{2}{|c|}{2.418} & \multicolumn{2}{|c|}{2.805} \\
\hline Potential & 5.9160 & \pm 0.0053 & 8.326 & \pm 0.024 \\
\hline$L_{\mathrm{y}}$ & 0.8319 & \pm 0.0005 & 0.1681 & \pm 0.0005 \\
\hline$L_{\mathrm{b}}$ & 0.8350 & \pm 0.0005 & 0.1650 & \pm 0.0005 \\
\hline$L_{\mathrm{v}}$ & 0.8374 & \pm 0.0005 & 0.1626 & \pm 0.0005 \\
\hline$L_{\mathrm{u}}$ & 0.8364 & \pm 0.0005 & 0.1636 & \pm 0.0005 \\
\hline $\log g$ [cgs] & 3.684 & & 4.137 & \\
\hline$M\left[M_{\odot}\right]$ & 12.00 & \pm 0.07 & 8.47 & \pm 0.10 \\
\hline$R\left[R_{\odot}\right]$ & 8.248 & \pm 0.014 & 4.118 & \pm 0.015 \\
\hline$M_{\mathrm{bol}}$ & -6.565 & & -4.764 & \\
\hline Synchr. ${ }^{* *} V_{\text {rot }} \sin i\left[\mathrm{~km} \mathrm{~s}^{-1}\right]$ & 66.2 & & 33.0 & \\
\hline Observ. $V_{\text {rot }} \sin i\left[\mathrm{~km} \mathrm{~s}^{-1}\right]$ & 160.0 & & 92.7 & \\
\hline
\end{tabular}

Notes. ${ }^{(*)}$ The synchronicity parameter $F_{j}=P_{\mathrm{orb}} / P_{\text {rot }, j}(j=1,2)$ of both components was kept fixed during each iteration, but it was recalculated for new radius and inclination after each iteration, using $V_{\text {rot }} \sin i$ values of $160 \mathrm{~km} \mathrm{~s}^{-1}$, and $93 \mathrm{~km} \mathrm{~s}^{-1}$ for the primary and secondary, respectively. ${ }^{(* *)}$ These velocities are the pseudosynchronization velocities (Hut 1981) calculated as $r_{i} \cdot\left(K_{1}+K_{2}\right) \times$ coeff. according to Hut (1981). The optimized parameters are given with only their formal errors derived from the covariance matrix.

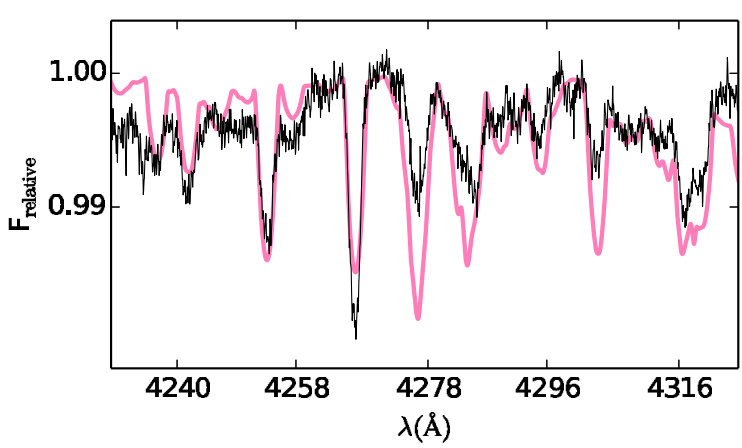

Fig. 5. Disentangled spectrum of the secondary in the wavelength region from 4230 to $4325 \AA$. The metallic lines seen in the observed spectrum are the O II lines at 4253.9, 4254.1, 4276.8, 4283.0 and $4303.8 \AA$, N II $4241.8 \AA$ line and C II $4267.1 \AA$ line. The O II lines in the disentangled spectrum of the secondary (black line) are weaker than suggested by the synthetic spectrum for the temperature of $25500 \mathrm{~K}$ (violet/gray line).

The GCHV light curves were solved by GCA, who used the program WINK (Wood 1973), and noted the disagreement between the theoretical and observed curves around periastron. Even the inclusion of the reflection did not improve the fit. In a "Note added in proof" GCA remarked that a more advanced treatment of the gravity darkening - in addition to other program changes - removed most of the disagreement, but they gave no numerical details.
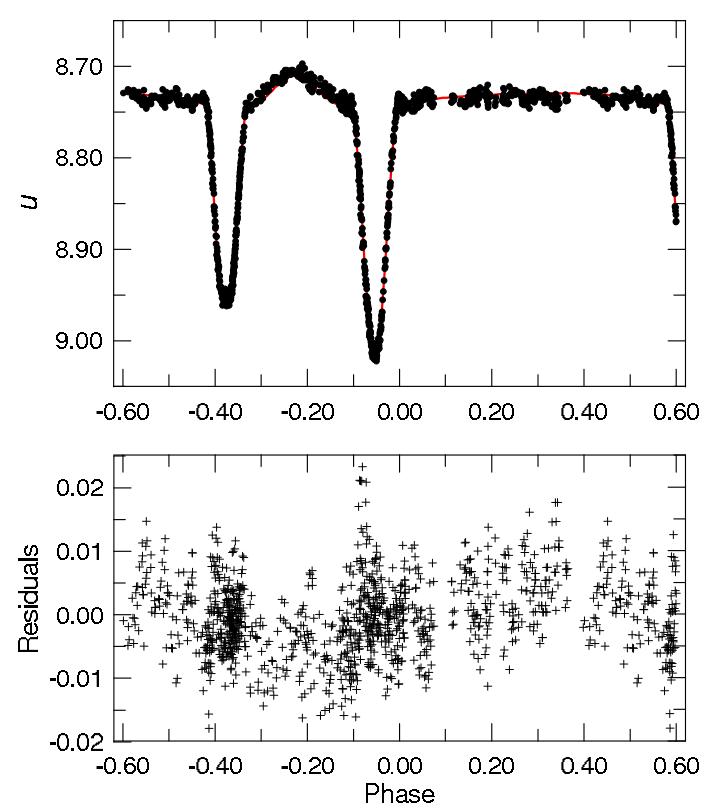

Fig. 6. Light curve of V346 Cen. Points correspond to the $u$ measurements by GCHV, the solid line to the solution by PHOEBE with parameters from Table 5. We show the $u$ data since their deviations from the model curve are the largest, as they are in GCA.

\section{Absolute parameters and apside line rotation}

The absolute parameters of the components are in Table 5. We compared these values with the evolutionary models by Claret (2004). 
We followed the steps used by Claret \& Giménez (2010) and calculated the coefficients $c_{2 i}$ (respecting the fast rotation of both components) and the general relativity contribution $(0.00130 \mathrm{deg} /$ cycle $)$. Then $\dot{\omega}_{\text {obs }}=0.02034, \dot{\omega}_{\text {newt }}=0.01904$, and $\log k_{2, \text { newt }}=-2.428$. To find the expected value of the apside line rotation, the tables published by Claret (2004) were considered. However, in this case the Claret models do not represent the primary component well. For the given mass and radius the model is too cold and old. From our parameters, the value of the primary $k_{2}$ might be estimated as -2.36 . The fast rotation probably means that the correct model value is smaller (Claret 1999), i.e. close to the observed value.

As the binary is a member of the open cluster Stock 14, it is possible to compare the distance obtained from the binary parameters with the distance determined for the cluster. Paunzen \& Netopil (2006) give the cluster distance as $2439 \pm$ 326 pc (according to Fitzgerald \& Miller 1983, the distance is $2870 \mathrm{pc}$ ) and the age $10 \mathrm{Myr}$. The parameters of the binary components obtained by GCA are very similar to ours; therefore, their results - age $10 \mathrm{Myr}$ and distance $2380 \mathrm{pc}$ - are still valid. The good agreement with the cluster data supports the correctness of the binary solution.

The only remaining open problem is to confirm the abrupt change in the orbital period and to find its true cause.

Acknowledgements. The research of P.M., P.H., M.W., and J.N. was supported by the grants P209/10/0715 and GA15-02112S of the Czech Science Foundation. Mr. D. Drobek kindly informed us about details of Siding Springs CCD observations of V346 Cen. We gratefully acknowledge the use of the electronic databases: SIMBAD at CDS, Strasbourg, and NASA/ADS, USA, and the data archive of Acta Astronomica. The operation of the robotic telescope FRAM has been supported by the grants of the Ministry of Education of the Czech Republic (MSMT-CR LG13007 and LG15014) and by grant No. 14-175 of the Czech Science Foundation.

\section{References}

Aller, L. H., Appenzeller, I., Baschek, B., et al. 1982, Landolt-Börnstein: Numerical Data and Functional Relationships in Science and Technology, New Series, Group 6 Astronomy and Astrophysics, Vol. 2 (Springer)

Brož, M., Mayer, P., Pribulla, T., et al. 2010, AJ, 139, 2258

Claret, A. 1999, A\&A, 350, 56

Claret, A. 2004, A\&A, 424, 919

Claret, A., \& Giménez, A. 2010, A\&A, 519, A57
Desmet, M., Frémat, Y., Baudin, F., et al. 2010, MNRAS, 401, 418

Drobek, D., Pigulski, A., Shobbrook, R. R., \& Narwid, A. 2010, Astron. Nachr., 331, 1077

Drobek, D., Pigulski, A., Shobbrook, R. R., \& Narwid, A. 2013, Acta Astron., 63, 339

Dugan, R. S., \& Wright, F. W. 1939, Contributions from the Princeton University Observatory, 19, 10

Ebr, J., Janeček, P., Prouza, M., et al. 2014, Rev. Mex. Astron. Astrofis. Conf. Ser., 45,114

Fitzgerald, M. P., \& Miller, M. L. 1983, PASP, 95, 361

Gaposchkin, S. 1953, Annals of Harvard College Observatory, 113, 67

Giménez, A., \& Bastero, M. 1995, Ap\&SS, 226, 99

Giménez, A., \& Garcia-Pelayo, J. M. 1983, Ap\&SS, 92, 203

Giménez, A., Clausen, J. V., \& Andersen, J. 1986a, A\&A, 160, 310

Giménez, A., Clausen, J. V., Helt, B. E., \& Vaz, L. P. R. 1986b, A\&AS, 66, 45

Hadrava, P. 2004a, Publ. Astron. Inst. Acad. Sci. Czech Rep., 92, 1

Hadrava, P. 2004b, Publ. Astron. Inst. Acad. Sci. Czech Rep., 92, 15

Harmanec, P. 1983, Hvar Observatory Bull., 7, 55

Harmanec, P. 1984, Bull. Astron. Inst. Czechoslovakia, 35, 193

Harmanec, P. 1998, A\&A, 335, 173

Harmanec, P., Matthews, J. M., Bozic, H., et al. 1991, Bull. Astron. Inst. Czechoslovakia, 42, 1

Harmanec, P., Koubský, P., Nemravová, J. A., et al. 2015, A\&A, 573, A107

Hernandez, C. A., \& Sahade, J. 1978, PASP, 90, 728

Hut, P. 1981, A\&A, 99, 126

Khaliullina, A. I., Khaliullin, K. F., \& Martynov, D. I. 1985, MNRAS, 216, 909

Lanz, T., \& Hubeny, I. 2007, ApJS, 169, 83

Lesh, J. R. 1968, ApJS, 17, 371

Mermilliod, J.-C., Mermilliod, M., \& Hauck, B. 1997, A\&AS, 124, 349

Mikuz, H., Dintinjana, B., Prsa, A., Munari, U., \& Zwitter, T. 2002, IBVS, 5338, 1

O'Connell, D. 1939, Publ. Riverview College Observatory, 2, 5

Ogloza, W., \& Zakrzewski, B. 2004, IBVS, 5507, 1

O’Leary, W., \& O'Connell, D. 1936, Astron. Nachr., 259, 399

Paunzen, E., \& Netopil, M. 2006, MNRAS, 371, 1641

Perryman, M. A. C., \& ESA 1997, The HiPPARCOs and TYCHO catalogues, Astrometric and photometric star catalogues derived from the ESA HIPPARcos Space Astrometry Mission (Noordwijk, The Netherlands: ESA Publ. Division)

Pigulski, A., \& Pojmański, G. 2008, A\&A, 477, 917

Pojmański, G. 1998, Acta Astron., 48, 35

Pojmański, G. 2000, Acta Astron., 50, 177

Pojmański, G. 2002, Acta Astron., 52, 397

Popper, D. M. 1966, AJ, 71, 175

Prša, A., \& Zwitter, T. 2005, ApJ, 628, 426

Prša, A., \& Zwitter, T. 2006, Ap\&SS, 36

van Houten, C. J., van Houten-Groeneveld, I., van Genderen, A. M., \& Kwee, K. 2009, J. Astron. Data, 15, 2

Wood, D. B. 1973, PASP, 85, 253

Zejda, M., Mikulášek, Z., \& Wolf, M. 2008, A\&A, 489, 321

Zucker, S., \& Mazeh, T. 1994, ApJ, 420, 806 

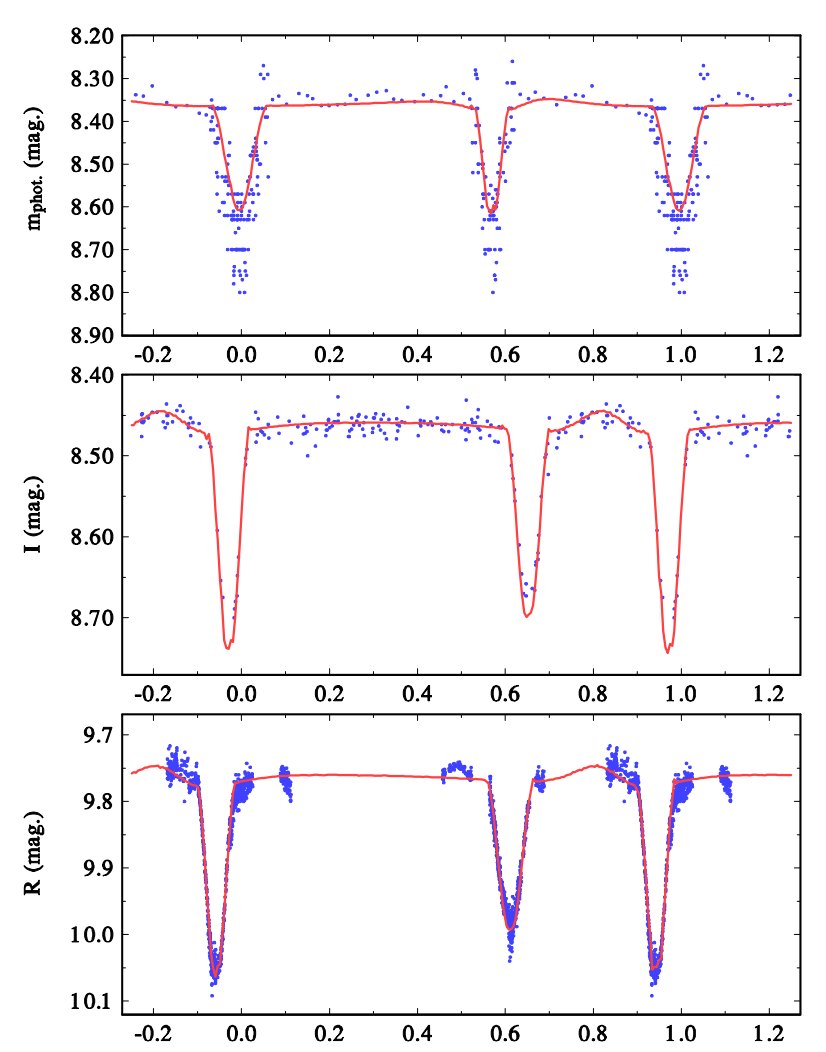

Fig. A.1. Examples of local fits in PHOEBE for selected light curves. From top to bottom: (1) photographic light curve by O'Connell (1939); (2) ASAS2 I light curve (RJDs 51 200-51 403); (3) $R$ light curve from FRAM automatic telescope.

\section{Appendix A: Photometric data used}

Since PHOEBE internally uses fluxes calculated from the observed magnitudes, normalized to some defined magnitude value, it is advisable to use observations converted at least approximately to magnitudes and not the magnitude differences alone, especially in situations when different comparison stars were used by different observers.

Here we provide some comments on individual data sets used for those who would like to carry out independent analyses. Unpublished individual magnitudes with their RJDs are available at CDS as Tables A.2 to A.6. The journal of all data sets is in Table 1. For illustration, we also show the quality of local fits for several different light curves in Fig. A.1.

- Dugan \& Wright (1939) Times of minima derived by us are in Table A.1.

- Riverview photographic magnitudes: these photographic observations were obtained by O'Connell (1939) and reduced from photographic plates relative to four comparison stars HD 101511, HD 101838, HD 101794, and HD 102053. We digitized the individual observations from both minima from their Table II. Unfortunately, observations outside minima were only published as normal points in Table III. For normal points outside minima we therefore reconstructed artificial RJDs, corresponding to the mean epoch of all observations, and assigned them weights proportional to the number of observations forming each normal point. This data set is also available at CDS as Table A.2.

- Walraven VBUL observations: these differential observations were published by van Houten et al. (2009) in the form of magnitude differences (not as the usual $\log [$ flux] values)
Table A.1. Times of the primary and secondary minima of V346 Cen estimated by us from Table 3 of Dugan \& Wright (1939).

\begin{tabular}{cccll}
\hline \hline $\begin{array}{c}\text { Min I } \\
\text { (RJD) }\end{array}$ & $w$ & $\begin{array}{c}\text { Min II } \\
\text { (RJD) }\end{array}$ & $w$ & Note \\
\hline 12673.377 & 0 & 11532.331 & 0 & not used \\
13653.191 & 1 & 13744.780 & 1 & primary min. not used \\
14127.347 & 1 & 14250.558 & 1 & primary min. not used \\
15486.954 & 1 & 15546.631 & 3 & \\
16561.646 & 2 & 16684.508 & 2 & \\
17509.889 & 2 & 17411.645 & 3 & \\
18236.908 & 2 & 18296.778 & 3 & \\
19596.136 & 2 & 19498.032 & 2 & \\
20512.745 & 3 & 20635.906 & 3 & \\
21397.778 & 3 & 21426.336 & 2 & \\
22283.020 & 2 & 22342.918 & 1 & \\
23357.561 & 1 & 23417.791 & 2 & \\
24400.886 & 2 & 24302.952 & 2 & \\
25570.370 & 1 & 26547.313 & 2 & \\
26455.380 & 2 & 27590.554 & 1 & \\
27087.549 & 1 & 28254.275 & 2 & \\
28288.834 & 1 & - & - & \\
\hline
\end{tabular}

Notes. See the text for details.

relative to the comparison star HD 101794 , for which we adopted $V=8^{\mathrm{m}} .684, B=8^{\mathrm{m}} .733$, and $U=7^{\mathrm{m}} \cdot 990$ from the electronic catalogue maintained at the Geneva Observatory (see Mermilliod et al. 1997), and an artifically estimated value $L=8^{\mathrm{m}} .300$, and added these values to the respective magnitude differences.

- ESO La Silla uvby observations: these superb photometric observations were published by Giménez et al. (1986b) as magnitude differences relative to HD 101466 . We adopted the values derived by these authors for HD $101466(y=$ $7^{\mathrm{m}} .322, b=7^{\mathrm{m}} \cdot 367, v=7^{\mathrm{m}} .535$, and $\left.u=8^{\mathrm{m}} .765\right)$ and added them to the magnitude differences. Since these observations are not available in digital form, we deposited them as Table A.3 at CDS.

- ASAS 2 I all-sky photometry: these observations were divided into four shorter time intervals not only to monitor the changes due to apsidal motion, but also because these early observations exhibit a secular drift. Data from the first time interval are also notably less accurate. We omitted a number of observations with larger deviations from the phase curve.

- ASAS $3 \mathrm{~V}$ all-sky photometry: these observations were divided into two shorter time intervals to monitor the changes due to apsidal motion. Data from diaphragm No. 3 were used because they have, on average, the smallest rms errors. We omitted a number of observations with larger deviations from the phase curve.

- Mount John BVR photometry: these differential CCD observations were secured by $\mathrm{MZ}$ relative to the Be star HD 101794 = V916 Cen, but it seems that the irregular variability of the comparison had no adverse effects on the photometry of V346 Cen, which was secured only during three consecutive nights. For comparison, we adopted $V=8 \mathrm{~m} \cdot 684$ and $B=8^{\mathrm{m}} .733$ from the Geneva database (Mermilliod et al. 1997), and artificially set $R=9 \mathrm{~m} .8$. The RJDs and magnitude differences V346 Cen - V916 Cen are available as Table A.4 at CDS. 
- Siding Springs UBV photometry: these observations were secured by Drobek et al. (2013) and are available in electronic form as magnitude differences to the mean of several comparison stars in the field of the CCD camera, but no details are known. We added $\Delta V=10^{\mathrm{m}} .66, \Delta B=10^{\mathrm{m}} .64$, and $\Delta U=10^{\mathrm{m}} .06$ to the magnitude differences to reproduce the $U B V$ magnitudes of V346 Cen.

- Sutherland broad-band green CCD photometry: these observations were obtained by JL during his visit to the South African Astronomical Observatory and were reduced relative to the A0 star HD $102152\left(V=9^{\mathrm{m}} .15\right.$, and $B=9^{\mathrm{m}} .16$ according to Mermilliod et al. 1997). We added $9^{\mathrm{m}} .15$ to the magnitude differences. The RJDs and magnitude differences V346 Cen - HD 102152 are available as Table A.5 at CDS.

- FRAM BVRI photometry: these CCD observations were secured by $\mathrm{JJ}, \mathrm{KH}$, and MM with the remotely controlled automatic photometric telescope located at the Pierre Auger Observatory in Argentina. They were reduced relative to CD-61 3157 , for which we adopted $V=9^{\mathrm{m}} .4, B=11^{\mathrm{m}} .2$, and $R=9^{\mathrm{m}} .900$ from the SIMBAD database. The RJDs and magnitude differences are in Table A.6 at CDS.

\section{Appendix B: Nature of the light variations of V916 Cen}

As already mentioned, the light variations of this object were discovered by Perryman \& ESA (1997) and classified - correctly, in our opinion - as typical for Be stars (see Fig. B.1). Harmanec (1983) already pointed out that Be stars vary on at least three distinct time scales, combining long-term changes due to secular variations of their extended envelopes, medium time changes on a scale of week to months, and rapid cyclic or even periodic changes occurring on a time scale comparable to stellar rotational period. Pojmański (2000) analysed the ASAS2 photometry secured in the infrared $I$ filter and reported that the star is an eclipsing binary with two similar eclipses, a period of $1 \mathrm{~d} 463279$, and a full amplitude of $0^{\mathrm{m}} .1$. Later Pigulski \& Pojmański (2008) analysed the ASAS3 V photometry and concluded that the object is also a $\beta$ Cep variable with a period of 0.224648 and an amplitude of 0.015 , and a $\lambda$ Eri variable with a period of 0.54362 and an amplitude of $0^{\mathrm{m}} .011$. After removing the rapid changes and long-term variations, they were able to fold the residuals with a period of

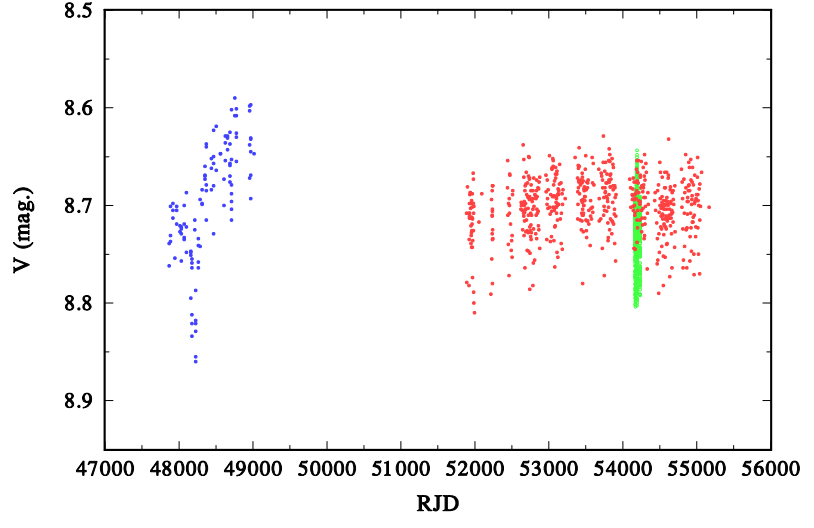

Fig. B.1. Time plot of the $V$ magnitude of V916 Cen = HD 101794 versus time. Blue dots are the HIPPARCos $H_{\mathrm{p}}$ observations (Perryman \& ESA 1997, flags 0 and 1 only) converted to the Johnson $V$ magnitude after Harmanec (1998), red dots are the ASAS3 observations (Pojmański 2002), and green dots are observations published by Drobek et al. (2013). Long-term changes of this B0.5e star are obvious.

1.4632361, which they again interpreted as the light curve of a double-lined eclipsing binary. Drobek et al. (2010) analysed their $V$ photometry from the Siding Springs Observatory and once more confirmed the 1.4632 period after removal of rapid changes for which they found a period of 0.22481 .

We inspected one HARPS ESO and two FEROS archival spectra of V916 Cen to find that they do not show evidence of large RV changes, expected for a short-period eclipsing binary. We estimated the projected rotational velocity as $v \sin i=$ $250 \mathrm{~km} \mathrm{~s}^{-1}$. We note that for a reasonable equatorial radius of the $\mathrm{B} 0$ star, e.g. $7 R_{\odot}$, the 1.46 is a plausible value for the stellar rotational period.

We therefore tentatively suggest that V916 Cen is not an eclipsing binary but a typical Be star also showing variations on the time scale of stellar rotation. Good examples of such light curves are EM Cep (see Harmanec 1984, $P=0$ d8) or LQ And (Harmanec et al. 1991, $P=$ 0d62). Particularly, EM Cep had long been considered to be an eclipsing binary, but no corresponding RV changes could be found for it or for LQ And. A dedicated spectral and photometric study of the object is undoubtedly still necessary. 